\title{
Diagnóstico y tratamiento inicial de la uveítis por médicos no oftalmólogos
}

\author{
Diagnosis and treatment of uveitis by non-ophthalmologist physicians
}

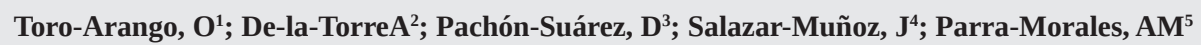

\section{Resumen}

Objetivos: Describir los principales signos y síntomas, así como el tratamiento inicial de un paciente con diagnóstico de uveítis con el propósito de evitar retardo en la remisión y el inicio del manejo oportuno de esta patología. Métodos: Se realizó una revisión sistemática de la literatura en la cual se identificaron los principales signos y síntomas que deben ser tenidos en cuenta para sospechar el diagnóstico de uveítis y su tratamiento indicado. Se llevó a cabo una búsqueda en PubMed durante el periodo del 2010-2015, se eligieron 439 artículos, los cuales fueron sometidos a dos procesos de filtrado manual, eligiendo por un grupo de investigadores los resúmenes que respondieran nuestra pregunta de investigación. Posteriormente un grupo de oftalmólogos expertos y médicos con experiencia en oftalmología realizaron una segunda revisión. Resultados: Posterior al primer proceso de filtrado se obtuvieron 73 artículos que se sometieron a un segundo proceso de selección donde se obtuvieron 30 artículos. De la selección final de artículos: seis fueron estudios observacionales o ensayos clínicos; los artículos restantes fueron revisiones de la literatura. Los estudios observacionales y ensayos clínicos aleatorizados se evaluaron con la herramienta GRADE siendo la mayoría de evidencia alta. Se hizo una revisión de los treinta artículos seleccionados, por parte de nuestros investigadores, dando como resultado recomendaciones para el diagnóstico y tratamiento de la uveítis para el personal médico no oftalmólogo. Conclusiones: Existen un conjunto de signos y síntomas que pueden hacer sospechar al médico no oftalmólogo el diagnóstico de uveítis. Ante un paciente que presenta dolor ocular asociado a disminución de la agudeza visual, fotofobia, ojo rojo sin secreción conjuntival, miosis pupilar, depósitos retroquératicos y hallazgos al fondo de ojo como vitreitis o coriorretinitis se debe sospechar una uveítis. El manejo precoz de la uveítis anterior no infecciosa puede ser instaurado por el médico no oftalmólogo con corticoides tópicos, sin embargo, en caso de uveítis intermedia y posterior es importante remitir al oftalmólogo cuanto antes. Proponemos un algoritmo para ayudar al diagnóstico de la uveítis y recomendaciones para su manejo inicial.

Palabras claves: Uveítis, Diagnóstico temprano, Manejo inicial, Corticoides, Ciclopléjicos.

\footnotetext{
1. Especialista en Oftalmología. Hospital Universitario Fundación Santa Fe. Docente Oftalmología, Universidad de Los Andes.

2. Especialista en Oftalmología. Subespecialista en Inmunología Ocular y Uveítis. Coordinadora Servicio Inmunología, Grupo NeUros,

Universidad del Rosario.

3. Especialista en Oftalmología Fundación Universitaria Sanitas. Subespecialista en Enfermedades Inflamatorias Oculares.

4. Especialista en Medicina Interna y Epidemiología. Hospital Universitario Fundación Santa Fe. Docente Universidad de los Andes y

Universidad del Bosque.

5. Médico Universidad de Los Andes.
} 


\begin{abstract}
Objective: The purpose of this study was to describe the main diagnostic features and the initial management of uveitis, in order to avoid delays in the treatment and the remission to the ophthalmologist. Methods: A systematic review of the literature, which identified the main signs and symptoms and initial treatment for patients with uveitis, was done. A search of the literature published through 2010-2015 was performed using PubMed database. Four hundred and thirty nine eligible articles underwent a selection process in which titles, abstracts and full texts were reviewed, obtaining thirty final articles. Results: Thirty articles were reviewed, six were observational studies and clinical trials; 24 articles were reviews or case reports. The observational studies and clinical trials were rated with the Grading of Recommendations Assessment (GRADE) tool. After the grading tree of the articles were of high level of evidence. Conclusions: In a patient with ocular pain related to decreased visual acuity, photophobia, red eye without secretion, miosis, keratic precipitates, and vitritis or chorioretinitis the healthcare provider should rule out the diagnosis of uveitis. A non-ophthalmologist physician can start the initial management of an anterior uveitis and then refer the patient to the ophthalmologist as soon as possible. If the diagnosis is an infectious anterior uveitis, intermediate uveitis or posterior uveitis the patient should be derived to the ophthalmologist immediately.
\end{abstract}

Keywords: Primary health care, uveitis, iridocyclitis, choroiditis, retinochoroiditis, diagnosis, therapy.

\section{Introducción}

La uveítis se define como la inflamación del tracto uveal y/o de sus estructuras adyacentes $(1,2)$. Esta patología se puede presentar como manifestación de una enfermedad sistémica, o formar parte de las uveítis idiopáticas $(1,3,4)$. La incidencia reportada de uveítis en países desarrollados varía entre 15 y 17 casos por cada 100.000 habitantes, con una prevalencia de $0,1 \%,(4,5)$. El pico de incidencia se encuentra entre los 20 y los 50 ańos de edad $(4,5)$. La forma anterior es la variedad de presentación más común y también la de menor severidad, representando aproximadamente un $90 \%$ de los casos (5). En Colombia existen escasos estudios que describen el patrón de distribución de la uveítis; sin embargo, los hallazgos concuerdan con la evidencia existente a nivel mundial (6).

El sistema inmunológico cumple un papel fundamental para el desarrollo de dicha respuesta inflamatoria, ya sea de origen infeccioso o autoinmune (7-10). Durante este proceso inflamatorio se ha visto la participación de diversas moléculas que contribuyen al desarrollo de la uveítis como son: las citoquinas, quimiocinas y moléculas de adhesión (11-13). A pesar del denominado privilegio inmune ocular la aparición de uveítis autoinmune sigue siendo común y su causa aún está por establecer $(14,15)$.

El diagnóstico es inicialmente clínico $(16,17)$ y la etiología en su mayoría es idiopática (16). No existe un consenso sobre pruebas de laboratorio o imágenes que deban ser realizadas para el diagnóstico y manejo inicial, así como el seguimiento para estos pacientes $(18,19)$. A pesar de esto, existen diferentes exámenes que pueden realizarse cuando la inflamación con localización anterior es moderada-severa, bilateral, recurrente, granulomatosa o cuando está asociada a síntomas sistémicos que sugieran enfermedad subyacente (20-23). Para descartar enfermedad por Treponema Pallidum se debe realizar VDRL y FTAabs. En el caso de Mycobacterium tuberculosis se recomienda una placa de tórax, una PPD y/o prueba de quantiferón, para identificar 
los pacientes expuestos al bacilo. Para enfermedades sistémicas, en el caso de las espondiloartropatías se debe realizar un HLA B27 y cuando se sospecha sarcoidosis el examen de elección es una radiografía de tórax y niveles séricos de enzima convertidora de angiotensina -ECA- o lisozima $(21,22)$.

En cuanto al tratamiento, el patrón de oro para el manejo de la uveítis no infecciosa continúa siendo el uso de corticoesteroides (24). Es importante antes de iniciar el tratamiento descartar o confirmar las causas infecciosas y tener en cuenta el síndrome de enmascaramiento por neoplasias, pues estos diagnósticos requieren tratamiento de la causa subyacente $(24,25)$.

Debido a los muchos efectos adversos del uso a largo plazo de corticoesteroides, han surgido nuevas terapias basadas en la inmunomodulación e inmunosupresión (25-27). Estos nuevos agentes (entre los cuales se destaca el adalimumab por ser el primer biológico aprobado por la FDA para tratar uveítis) son prometedores para el manejo de la uveítis y están siendo evaluados actualmente $(25,28,29)$.

\section{Metodología}

\section{Tipo de estudio}

Se plantea una revisión sistemática con el objetivo de describir los principales signos y síntomas que deben ser tenidos en cuenta por el médico no oftalmólogo ante la sospecha de uveítis, y definir el tratamiento inicial.

La búsqueda bibliográfica se realizó en las bases de datos Medline (PubMed). La estrategia de búsqueda de artículos sobre los signos y sintomas de uveitis fue la siguiente: "uveitis" OR "iridocyclitis" OR "choroiditis" AND "primary health care" OR "uveitis" OR "iridocyclitis" OR "choroiditis" AND "diagnosis" OR "uveitis" OR “iridocyclitis" OR "choroiditis" AND "therapy" AND "Case Reports" OR "Clinical Trial" OR "Clinical Trial, Phase I" OR "Clinical Trial, Phase II" OR "Comparative
Study" OR "Controlled Clinical Trial" OR "Journal Article" OR "Consensus Development Conference" OR "Guideline" OR "Practice Guideline" OR "Review" OR "systematic" OR "Randomized Controlled Trial" OR "Observational Study" OR "Multicenter Study" OR "Meta-Analysis" OR "Introductory Journal Article" OR "Historical Article" AND "full text" AND "last 5 years" AND "Human" AND "English" OR "Spanish".

Periodo de tiempo: Desde enero 2010 hasta diciembre de 2015.

\section{Estrategia de búsqueda}

La estrategia de búsqueda se estableció con las palabras clave que identifican a la población y la intervención de interés. Se utilizaron combinaciones y restricciones según el motor de búsqueda. Se obtuvieron 439 artículos sobre los cuales se realizó una primera estrategia de selección por parte de los investigadores donde, con base en título y resumen, se descartaron los artículos que no respondían la pregunta de investigación, obteniendo 73 artículos. Posteriormente se realizó una segunda estrategia de selección llevada a cabo por un oftalmólogo y un médico con experiencia en oftalmología donde se analizó el texto completo de los artículos seleccionando los que cumplían con los criterios de inclusión. Un tercer evaluador conocedor del protocolo resolvió las discrepancias. Los datos se extrajeron según un formato pre-establecido. Se descartaron los diferentes estudios por no ser la población de estudio, el diseño de interés, tipo de intervención o evaluar otro tipo de desenlaces de interés. Se seleccionaron los estudios de texto completo. Dos autores de forma independiente priorizaron los desenlaces acordes al sistema GRADE y realizaron una serie de recomendaciones para el personal no oftalmólogo.

\section{Criterios de selección}

\section{Criterios de Inclusión}

- Artículos de tipo reportes de casos, estudios clínicos, guías de manejo, revisiones sistemáticas, 
estudios observacionales, estudios multicéntricos, cuyo propósito fuera aportar en el diagnóstico y tratamiento de uveítis, iridociclitis y coroiditis, y que al ser evaluados con el sistema GRADE fueran adecuados para concluir cuál debe ser el manejo inicial por un médico no oftalmólogo de un paciente con diagnóstico de uveítis.

\section{Criterios de Exclusión}

- Estudios realizados en animales

- Estudios publicados únicamente como resúmenes

- Artículos en un idioma diferente al inglés

\section{Evaluación del riesgo de sesgo}

Se utilizaron los criterios descritos en "Cochrane Handbook of Sistematics Reviews", versión 5.1.0, para evaluar el riesgo de sesgos. Nos enfocamos en la valoración de los siguientes sesgos: sesgo de selección (generación de la secuencia y ocultación de la asignación), sesgo de realización (cegamiento de los participantes y del personal), sesgo de detección (cegamiento de los evaluadores de resultados), sesgo de desgaste (datos de resultados incompletos) y sesgo de notificación (notificación selectiva de los resultados.) La fortaleza de la evidencia se evaluó utilizando el sistema GRADE. Dos autores de forma independiente evaluaron el riesgo de sesgo.

\section{Aspectos éticos}

Este estudio se efectuó de conformidad con las normas éticas establecidas por la Declaración de Helsinki, acogiéndose a la legislación colombiana vigente, en el Parágrafo A del Artículo 11 de la Resolución 8430 de 1993 expedida por el Ministerio de Salud. Esta investigación es clasificada como "Investigación sin Riesgo", debido a que emplea un método de investigación documental secundaria de la literatura retrospectiva, de artículos ya publicados y no se realiza intervención o modificación intencionada de las variables biológicas, fisiológicas, sicológicas o sociales de individuos.

\section{Resultados}

La búsqueda en la base de datos de PubMed arrojó 439 resultados, los artículos fueron examinados por título y resumen. Se realizó una revisión completa de los documentos que parecían cumplir con los criterios de inclusión. Posteriormente, se hizo una selección manual por el equipo de investigación obteniendo 72 artículos que fueron sometidos a un segundo proceso de filtrado por un oftalmólogo experto y un médico con experiencia en oftalmología. Finalmente se obtuvieron 30 artículos que respondían a nuestra pregunta de investigación, de los cuales 2 artículos eran reportes de caso, 20 artículos revisiones, 4 estudios observacionales y 4 ensayos clínicos aleatorizados (ver Figura 1).

Todos los artículos fueron publicados en revistas indexadas entre la cuales se destacaron International Ophthalmology Clinics y Ophthalmology, con 4 y 3 artículos respectivamente. De los artículos seleccionados, 6 fueron aptos para evaluación mediante la herramienta GRADE, 3 artículos tuvieron nivel de evidencia alta, un artículo moderada y 2 artículos baja (ver Tabla 1).

En cuanto al enfoque de cada artículo, 15 se enfocaban en uveítis no infecciosa, 7 en uveítis infecciosa y 8 no lo especificaban. En todos los artículos se recomendaba el manejo con corticoides tópicos para la uveítis no infecciosa anterior, recordando que su uso a largo plazo puede generar efectos adversos $(24,25,27,30-33)$. Entre los artículos se mencionaban las nuevas tendencias para el manejo de uveítis que incluyen: alquilantes, antimetabolitos y agentes biológicos $(25,29,34,35)$.

En la uveítis infecciosa el tratamiento del agente causal era la recomendación general evitando el uso de corticoesteroides por riesgo de entorpecer la evolución clínica del paciente (36-38). 


\section{Búsqueda de artículos relevantes} con combinación de términos MeSH

en la base de datos PUBMED

$(n=439)$

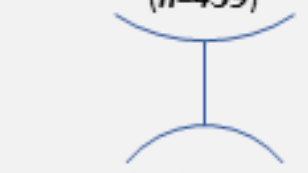

Lectura de Título y resumen de los artículos

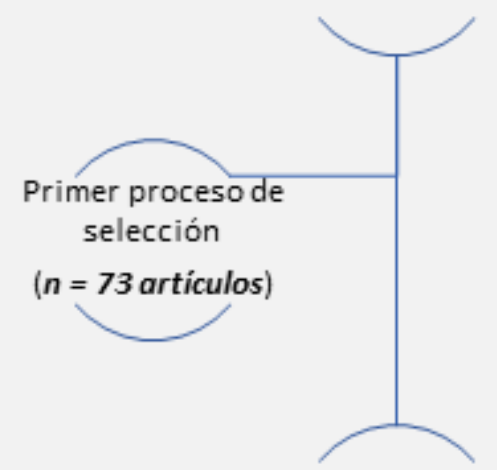

Lectura de texto completo

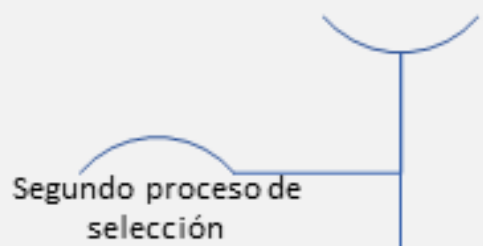
selección

( $n=30$ artículos)<smiles>C1CC1</smiles>

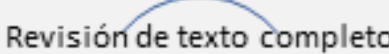
de los 30 artículos finales por dos investigadores independientes

\section{Reportes de caso $=2$}

Revisiones de la literatura $\mathbf{2 0}$

Estudios observacionales $=4$

Ensayos Clínicos aleatorizados $=4$

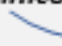

Redacción de

recomendaciones y

conclusiones

Figura 1. Búsqueda y selección de resultados. 
Tabla 1. Artículos Evaluados con Herramienta Grade

\begin{tabular}{|c|c|c|c|c|}
\hline Nombre & Autor & Año & Revista & $\begin{array}{l}\text { Tipo de } \\
\text { estudio }\end{array}$ \\
\hline $\begin{array}{l}\text { Clinical and epidemiological characteristics of patients with } \\
\text { uveitis in an emergency eye care center in Brazil. }\end{array}$ & $\begin{array}{l}\text { Camilo EN (1), } \\
\text { Moura GL (1), } \\
\text { Arantes TE(1). }\end{array}$ & 2013 & Arq Bras Oftalmol & $\begin{array}{r}\text { Estudio } \\
\text { observacional }\end{array}$ \\
\hline $\begin{array}{l}\text { Difluprednate } 0.05 \% \text { versus prednisolone acetate } 1 \% \\
\text { for endogenous anterior uveitis: a phase III, multicenter, } \\
\text { randomized study. }\end{array}$ & $\begin{array}{l}\text { John D. Sheppard, } \\
\text { Melissa M. Toyos, } \\
\text { John H. Kempen, } \\
\text { Paramjit Kaur, and } \\
\text { C. Stephen Foster }\end{array}$ & 2014 & $\begin{array}{r}\text { Invest Ophthalmol } \\
\text { Vis Sci }\end{array}$ & $\begin{array}{r}\text { Ensayo } \\
\text { clínico } \\
\text { aleatorizado }\end{array}$ \\
\hline $\begin{array}{l}\text { Comparison of efficacy and ocular surface toxicity of } \\
\text { topical preservative-free methylprednisolone and preserved } \\
\text { prednisolone in the treatment of acute anterior uveitis. }\end{array}$ & $\begin{array}{r}\text { Hedayatfar A(1), } \\
\text { Hashemi H, Asgari } \\
\text { S, Chee SP. }\end{array}$ & 2014 & Cornea. & $\begin{array}{r}\text { Ensayo } \\
\text { clínico } \\
\text { aleatorizado }\end{array}$ \\
\hline $\begin{array}{l}\text { Evaluation of dexamethasone phosphate delivered by ocular } \\
\text { iontophoresis for treating noninfectious anterior uveitis. }\end{array}$ & $\begin{array}{r}\text { Cohen AE (1), } \\
\text { Assang C, Patane } \\
\text { MA, From S, } \\
\text { Korenfeld M; } \\
\text { Avion Study } \\
\text { Investigators. }\end{array}$ & 2011 & Ophthalmology & $\begin{array}{r}\text { Ensayo } \\
\text { clínico } \\
\text { aleatorizado }\end{array}$ \\
\hline $\begin{array}{l}\text { Randomized comparison of systemic anti-inflammatory } \\
\text { therapy versus fluocinolone acetonide implant for } \\
\text { intermediate, posterior, and panuveitis: the multicenter } \\
\text { uveitis steroid treatment trial. }\end{array}$ & $\begin{array}{r}\text { Multicenter Uveitis } \\
\text { Steroid Treatment } \\
\text { (MUST) Trial } \\
\text { Research Group(1), } \\
\text { Kempen JH, } \\
\text { Altaweel MM, } \\
\text { Holbrook JT, Jabs } \\
\text { DA, Louis TA, } \\
\text { Sugar EA, Thorne } \\
\text { JE. }\end{array}$ & 2011 & Ophthalmology & $\begin{array}{r}\text { Ensayo } \\
\text { clínico } \\
\text { aleatorizado }\end{array}$ \\
\hline The causes of uveitis in a referral centre of Northern Italy. & $\begin{array}{r}\text { Cimino L(1), } \\
\text { Aldigeri R, } \\
\text { Salvarani C, Zotti } \\
\text { CA, Boiardi L, } \\
\text { Parmeggiani } \\
\text { M, Casali B, } \\
\text { Cappuccini L. }\end{array}$ & 2010 & $\begin{array}{r}\text { Int Ophthalmol } \\
\text { Clin }\end{array}$ & $\begin{array}{r}\text { Estudio } \\
\text { observacional }\end{array}$ \\
\hline
\end{tabular}




\begin{tabular}{|c|c|c|c|}
\hline Evento & Diagnóstico & Intervención & GRADE \\
\hline Uveítis & $\begin{array}{l}\text { Dolor ocular } \\
\text { - Ojo Rojo } \\
\text { - Visión Borrosa } \\
\text { - Fotofobia } \\
\text { - Hiperemia conjuntival } \\
\text { - Precipitados queráticos finos } \\
\text { - Sinequias posteriores } \\
\text { - Células en cámara anterior }\end{array}$ & No especifica & Baja \\
\hline $\begin{array}{l}\text { Uveítis } \\
\text { autoinmune }\end{array}$ & $\begin{array}{l}\text { - Células en cámara anterior : al menos } 11 \\
\text { - Flare: al menos grado II }\end{array}$ & $\begin{array}{r}\text { Recibir difluprednato } \\
0.05 \%(\mathrm{n}=56) 4 \text { veces } \\
\text { al día alternado con } \\
\text { vehículo } 4 \text { veces al día VS } \\
\text { Prednisolona acetato } 1 \% \\
(\mathrm{n}=54) \text { ocho veces al día. } \\
\text { Se realizó manejo durante } \\
14 \text { días. Se observó a los } \\
\text { pacientes por } 14 \text { días. }\end{array}$ & Moderada \\
\hline $\begin{array}{l}\text { Uveítis } \\
\text { anterior no } \\
\text { infecciosa }\end{array}$ & $\begin{array}{l}\text { - Células en cámara anterior : por lo menos } 2+\text { evidencia negativa de } \\
\text { inflamación infecciosa (Cicatrices corneales, atrofia de iris) o granulomatosa } \\
\text { (nódulos en iris o precipitados queráticos granulomatosos) }\end{array}$ & $\begin{array}{r}\text { Recibieron succinato de } \\
\text { metilprednisolona } 1 \% \text { sin } \\
\text { preservantes cada hora o } \\
\text { acetato de Prednisolona } \\
1 \% \text { con preservantes con } \\
\text { régimen de reducción cada } \\
2 \text { semanas }\end{array}$ & Alto \\
\hline $\begin{array}{l}\text { Uveítis } \\
\text { anterior no } \\
\text { infecciosa }\end{array}$ & - Conteo de células en cámara anterior mayor a 11 & $\begin{array}{r}\text { Fosfato de dexametasona } \\
\text { por iontoforesis a } \\
\text { diferentes dosis }\end{array}$ & Alto \\
\hline $\begin{array}{l}\text { Uveítis no } \\
\text { infecciosa }\end{array}$ & No especificado & $\begin{array}{r}\text { Terapia sistémica con } \\
\text { corticoesteroides VS } \\
\text { Implante intravitreo de } \\
\text { fluocinolona }\end{array}$ & Alto \\
\hline Uveítis & $\begin{array}{l}\text { - Toxoplasmosis:retinocoroiditis } \\
\text { - Sarcoidosis: hallazgos clínicos, biopsia transbronquial o con } 1 \text { de los } \\
\text { siguientes (ECA elevada, anergia cutánea, Radiografía Tórax positiva) } \\
\text { - Pars planitis idiopática (snowbank) } \\
\text { - Uveítis herpética inflamación intraocular aguda unilateral granulomatosa } \\
\text { con sinequias y atrofia sectorial del iris } \\
\text { - Behcet: ulceras orales más dos de los siguientes (ulceras genitales } \\
\text { recurrentes, lesión en ojo, lesión en pie, test de patergia positivo) } \\
\text { - Uveítis TB: uveítis granulomatosa PPD > 15; radiografía de tórax. } \\
\text { - Fuchs: Precipitados queráticos granulomatosos estrellados, iris atrófico }\end{array}$ & No especifican & Baja \\
\hline
\end{tabular}




\section{Discusión}

El análisis de los estudios seleccionados luego de nuestra búsqueda nos permite realizar recomendaciones para el diagnóstico y manejo de la uveítis dirigida al personal médico no oftalmólogo: una vez realizado el diagnóstico con base en las características clínicas, se deben tener en cuenta las causas y la clasificación de la uveítis.

La uveítis puede ser idiopática, infecciosa, efecto adverso de toxinas o la manifestación de un desorden autoinmune (39). En nuestra revisión, las uveítis idiopáticas y autoinmunes se incluían en el grupo de las uveítis no infecciosas. Un estudio observacional de Brasil que describió las características clínicas y epidemiológicas de pacientes con uveítis en el país, mostró concordancia con la literatura ya existente, siendo la uveítis idiopática la más frecuente, seguida de causas autoinmunes y en la minoría de los casos infecciosas. En cuanto a la clasificación anatómica, el estudio concluyó que la uveítis anterior es la más común y la que con mayor frecuencia se diagnóstica en los centros de atención primaria (40). La información anterior concuerda con el estudio realizado en nuestro medio, en el cuál se concluye también que la uveítis anterior es la más común (6).

La uveítis se divide en anterior, intermedia y posterior (41). En la bibliografía revisada existen recomendaciones similares en cuanto al diagnóstico de la uveítis; sin embargo, no hay consenso específico de criterios diagnósticos en ninguno de los artículos revisados.

La uveítis anterior se presenta con dolor ocular, con o sin visión borrosa, fotofobia, ojo rojo, inyección ciliar,células en cámara anterior, precipitados queráticos finos (ver Figura 2 y 3 ) o gruesos, nódulos en iris, fibrina, sinequias posteriores (ver Figura 5), flareo tyndall (ver Figura 6) e incluso hipopion $(41,42)$. Algunos artículos especifican que el número de células debe ser mayor a 2 células por campo y al menos de 11 células por campo (30,43-46), sin embargo, teniendo en cuenta que un reducido número de centros no especializados en oftalmología tiene acceso a lámparas de hendidura para el diagnóstico, el número de células por campo no sería de relevancia para nuestras recomendaciones. De hecho proponemos basarse en la opacidad en cámara anterior con el oftalmoscopio directo (ver anexos).

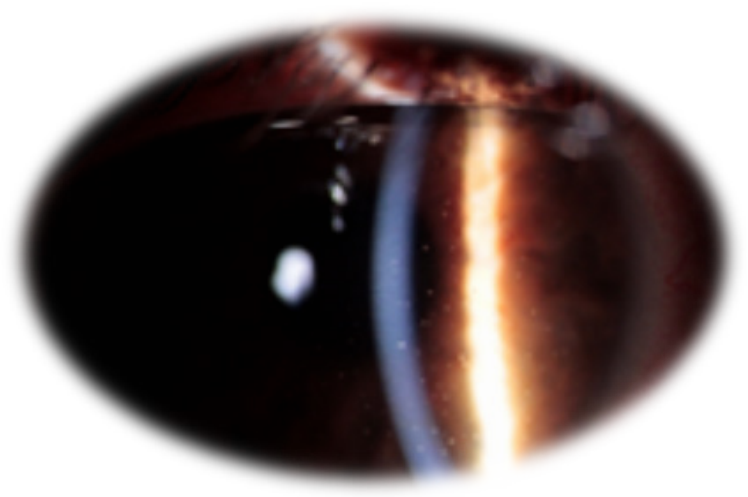

Figura 2. Precipitados retroquératicos finos en uveítis anterior. Cortesía Dra. Alejandra De La Torre.

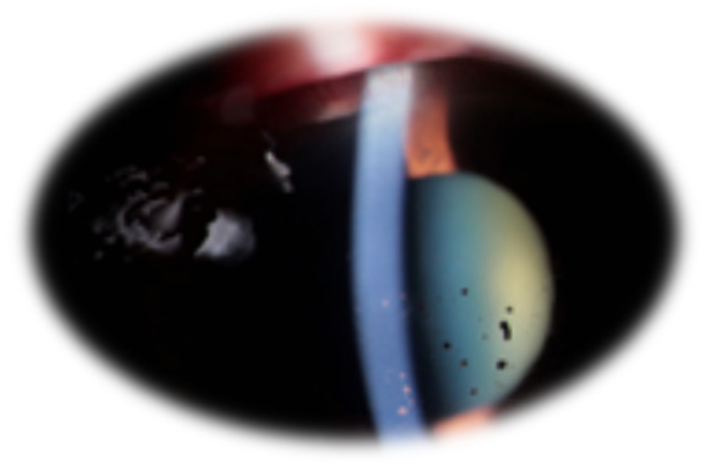

Figura 3. Precipitados retroquératicos finos pigmentados en uveítis anterior. Cortesía Dra. Alejandra De La Torre.

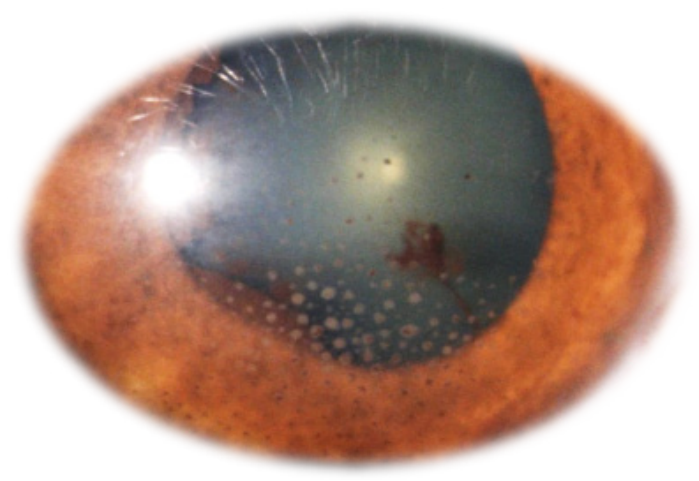

Figura 4. Precipitados retroqueráticos gruesos en uveítis anterior. Cortesía Dra Diana Pachón. 


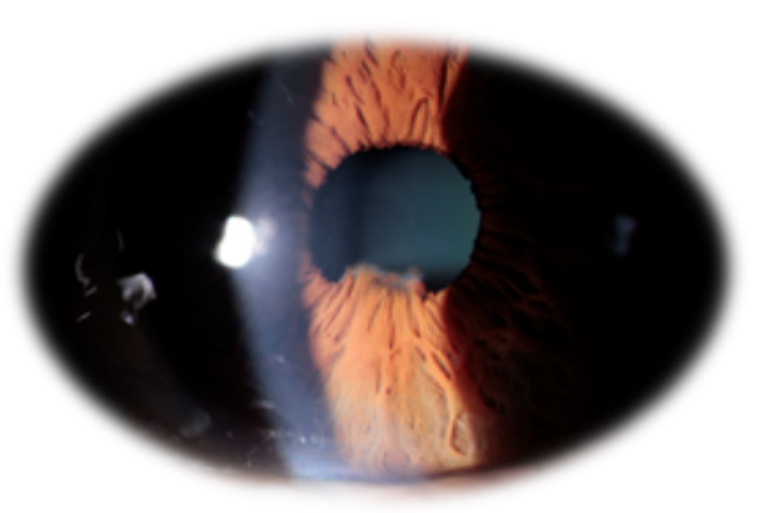

Figura 5. Sinequias posteriores. Cortesía Dra. Alejandra De La Torre.

La uveítis intermedia se caracteriza por visión borrosa, miodesopsias, vitreitis, bancos de nieve y bolas de nieve $(40,41)$; mientras que la uveítis posterior se caracteriza por presentar un cuadro clínico de miodesopsias o visón borrosa indolora asociado a lesiones en el fondo de ojo como, periflebitis, vitreitis o condensaciones vítreas (figura 7 y 8), lesiones algodonosas o cicatriciales, edema macular y papilitis $(36,40,47)$, puede cursar también conopacidades vítreas, coriorretinitis (ver Figura 9 y 10), retinitis, "snowballs" y desprendimiento exudativo de retina $(37,40,41)$.

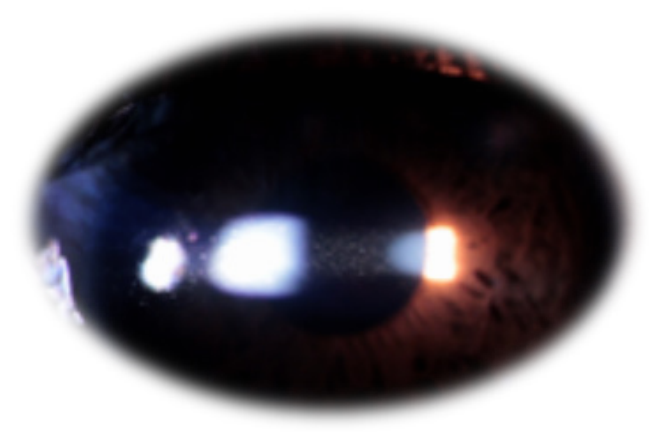

Figura 6. Tyndall. Cortesía Dra. Alejandra De La Torre.

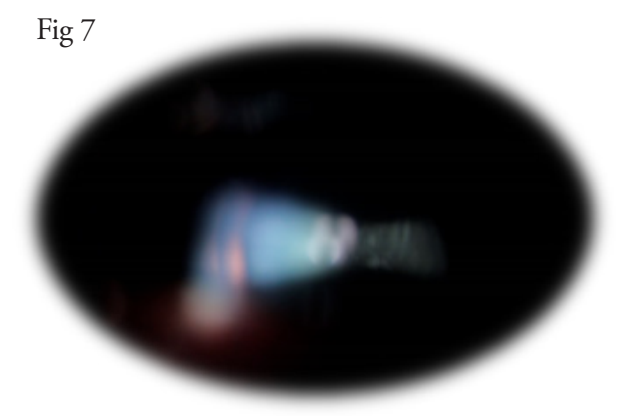

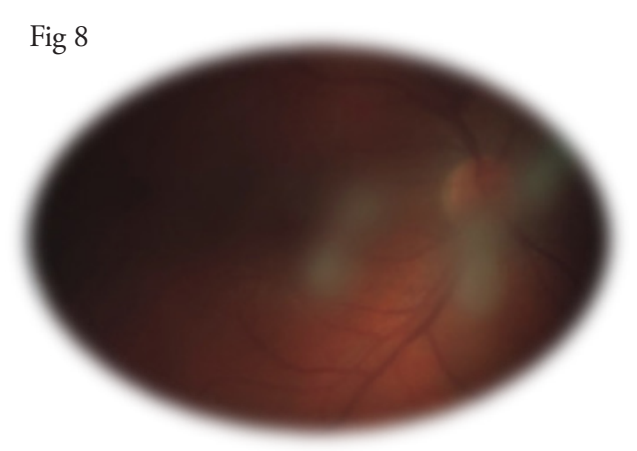

Figura 7 y 8. Vitreítis y condensaciones. Cortesía Dra. Alejandra de la Torre y Dra. Diana Pachón.
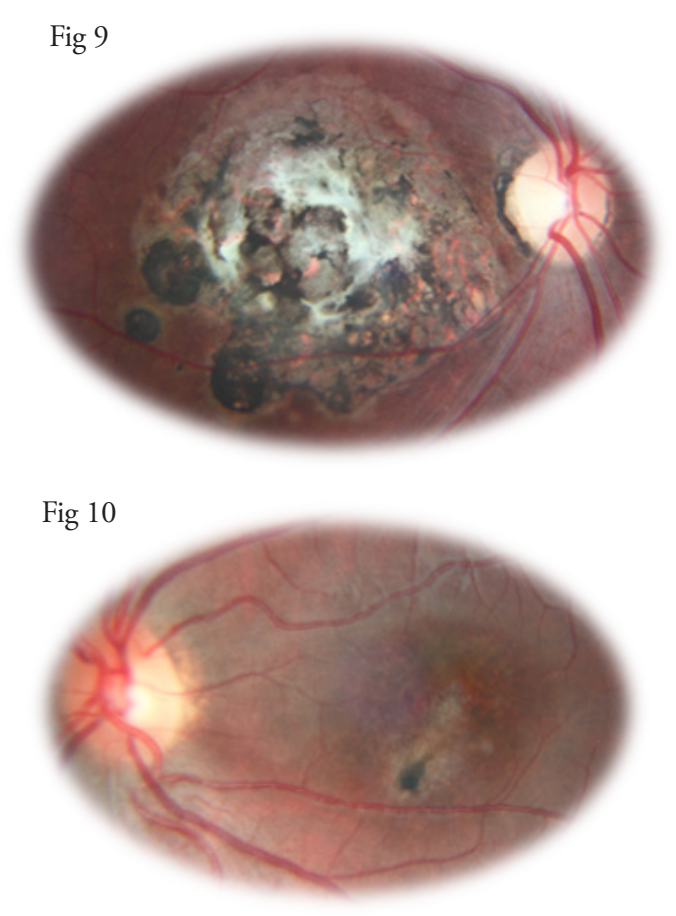

Figura 9 y 10. Coriorretinitis por Toxoplasmosis. Cortesía Dra. Alejandra de La Torre.

Uno de los diagnósticos diferenciales más importantes que debe tener en cuenta el médico al diagnosticar una uveítis es clasificar si es infecciosa o no. Esto es determinante a la hora de iniciar el tratamiento. Se debe realizar una historia clínica detallada en la cual se indagará por enfermedades autoinmunes (46), enfermedades sexualmente transmisibles, tuberculosis, exposición a carnes crudas, animales o aguas contaminadas; en pacientes con vesículas perioculares, dendritas en córnea e hipoestesia 
corneal asociadas signos y síntomas sugestivos de uveítis debe sospecharse herpes oftálmico $(37,48)$. En nuestro medio es importante también descartar asociación con enfermedades tropicales como lo son el Chikungunya y el dengue, dado que se han documentado como causa de uveítis, siempre que se sospeche alguna de las anteriores debe realizarse la serología específica para guiar el diagnóstico etiológico de la uveítis (ver Tabla 2)(47).

La uveítis no infecciosa es una de las presentaciones extrarticulares más frecuentes de la artritis seronegativa y es parte de la clasificación diagnóstica en la espondiloartritis seronegativa (espondilitis anquilosante, artropatía psoriásica, artritis enteropática y el Síndrome de Reiter)(46). La uveítis anterior, en un tercio de los pacientes, se asocia con espondiloartritis y a la presencia de HLA B27. La uveítis posterior es más frecuentemente diagnosticada en asociación con la enfermedad de Behcet o vasculitis de pequeños vasos HLA B51 (46). Las uveítis que se relacionan con un proceso autoinmune generalmente se presentan de manera unilateral y aguda, aunque puede volverse crónica en algunos casos (46).

Tabla 2. Ayudas diagnósticas para pacientes con sospecha de Uveítis.

\begin{tabular}{|l|l|}
\hline Agente & Serología \\
\hline Dengue & $\begin{array}{l}\text { IgM, Cuadro hemático con } \\
\text { plaquetas < a 50mil }\end{array}$ \\
\hline Chikungunya & IgM \\
\hline Toxoplasmosis & IgM, PCR, ELISA \\
\hline Sífilis & VDRL o RPR, T-Pa, FTAbs \\
\hline Tuberculosis & $\begin{array}{l}\text { Prueba de mantoux, } \\
\text { Quantiferón, Radiografía de } \\
\text { tórax }\end{array}$ \\
\hline Sarcoidosis & $\begin{array}{l}\text { Enzima convertidora } \\
\text { de angiotensina (ECA), } \\
\text { Radiografía de tórax }\end{array}$ \\
\hline Espondilitis anquilosante & HLA B27 \\
\hline $\begin{array}{l}\text { Artritis, Lupus eritematoso } \\
\text { sistémico }\end{array}$ & $\begin{array}{l}\text { Perfil autoinmune: Factor } \\
\text { reumatoideo, ANAS, } \\
\text { ANCAS }\end{array}$ \\
\hline
\end{tabular}

La importancia de descartar la uveítis infecciosa radica en el manejo de esta entidad frente a las uveítis de etiología no infecciosa. En los casos de uveítis infecciosa se deberá tratar el agente causal, el tratamiento debe ser iniciado idealmente por un equipo multidisciplinario que incluya Infectología y Oftalmología. Es importante no iniciar manejo con corticoesteroides, pues pueden entorpecer la evolución del paciente y empeorar el cuadro infeccioso. El médico no oftalmólogo puede dar manejo con ciclopléjicos (para prevenir la formación de sinequias) y analgesia (37).

El estándar para tratamiento de las uveítis no infecciosas se ha basado por años en el uso de corticoesteroides (24). Nuevas terapias han sido introducidas para evitar los efectos adversos a largo plazo de este tipo de medicamentos, entre los cuales se destacan la elevación de la presión intraocular, la catarata y el glaucoma (31). Los agentes biológicos, alquilantes y antimetabolitos son las terapias innovadoras que se han propuesto para el manejo de las uveítis no infecciosas $(31,33,49)$; aun así, los esteroides siguen siendo la recomendación para el manejo precoz de la uveítis no infecciosa $(27,31,35,45,49)$.

La vía de administración varía según la localización anatómica de la inflamación, siendo recomendados los esteroides tópicos para la uveítis anterior no infecciosa pero no para la intermedia o posterior pues el corticoesteroide tópico no alcanza niveles terapéuticos suficientes en la zona posterior del globo ocular $(32,50)$. En nuestro medio tenemos disponibles dos esteroides tópicos potentes: prednisolona acetato al $1 \%$ y dexametasona alcohol $0,1 \%$, aunque la dexametasona es más potente, la prednisolona acetato atraviesa mejor la capa lipídica de la córnea y de los tejidos por lo cual la recomendamos como primera línea de elección en el tratamiento de adultos y pacientes pediátricos (24,51-56).

La pauta sugerida para el manejo de uveítis anterior seria la siguiente:

- Uveítis no infecciosa en pacientes pediátricos: Prednisolona acetato al 1\% (ver Tabla 3) (52).

- Uveítis no infecciosa en pacientes adultos:

Prednisolona acetato 1\% (ver Tabla 3)(24). 
Asociado al inicio de los corticoesteroides tópicos es importante formular analgésicos y cicloplejia para manejo de dolor y prevención de formación de sinequias (ver Tabla 3)(50).

Tabla 3. Tratamiento precoz de uveítis anterior no infecciosa.

\begin{tabular}{|c|c|}
\hline Tratamiento & Posología \\
\hline $\begin{array}{l}\text { Acetato de } \\
\text { Prednisolona al } 1 \%\end{array}$ & $\begin{array}{l}\text { Adultos: } 1 \text { gota cada } 10 \text { minutos } \\
\text { por } 1-2 \text { horas, posteriormente } \\
\text { una gota cada hora por } 1-2 \text { días, } \\
\text { durante las horas de vigilia, luego } \\
\text { disminuir progresivamente la } \\
\text { dosis de } 1 \text { gota } 8 \text { veces al día hasta } \\
\text { aplicar una gota } 3 \text { veces al día } \\
\text { dependiendo de la severidad y de } \\
\text { la respuesta (ver anexo). } \\
\text { Pediátricos: Prednisolona acetato } \\
\text { al } 1 \% \text { una gota cada } 1-2 \text { horas } \\
\text { mientras está despierto y } 1 \text { dosis } \\
\text { de carga al despertar y antes de } \\
\text { dormir } 1 \text { gota cada } 5 \text { minutos } \\
\text { por } 5 \text { veces, dependiendo de la } \\
\text { severidad de la uveítis. }\end{array}$ \\
\hline Tropicamida $0,51 \%$ & 1 gota cada 8 horas \\
\hline Acetaminofén & 1 gramo cada 6 horas \\
\hline AINES & \\
\hline
\end{tabular}

En cuanto a la uveítis no infecciosa intermedia y posterior la recomendación son esteroides perioculares, intravítreos o sistémicos. Dichos tratamientos solo pueden ser administrados por un oftalmólogo en conjunto con el reumatólogo. Estos pacientes deben ser derivados a los especialistas para un manejo oportuno (31) .

Teniendo en cuenta lo anterior hemos diseñado un algoritmo y las guías del SUN modificadas (ver anexo), para facilitar el diagnóstico de las uveítis para el médico no oftalmólogo, sin necesidad de recurrir a equipos sofisticados.

\section{Conclusión y opinión de expertos}

Existen un conjunto de signos y síntomas que pueden hacer sospechar al médico no oftalmólogo el diagnóstico de uveítis. No es necesario el uso de equipos especializados para el diagnóstico de la uveítis anterior, un buen examen oftalmológico básico que incluya agudeza visual, examen externo, respuesta pupilar y fundoscopia directa es suficiente para diagnosticar esta entidad. Ante la presencia de dolor ocular asociado a fotofobia, ojo rojo, miosis pupilar, anisocoria, sin secreción conjuntival, depósitos retroquératicos con o sin disminución de agudeza visual, el paciente muy probablemente está cursando con una uveítis anterior. Lo primero que debe hacer el médico no oftalmólogo ante esta sospecha es descartar causas infecciosas y autoinmunes por medio de laboratorios diagnósticos. Una vez descartadas las causas infecciosas el manejo de la uveítis anterior puede ser instaurado por el médico con corticoesteroides tópicos, ciclopléjicos y analgesia. Los pacientes con disminución de la agudeza visual, miodesopsias, opacidades vítreas, con lesiones tipo manchas blanco-amarillentas en retina, coriorretinitis, papilitis y/o periflebitis (observadas en la oftalmoscopia directa) tienen un diagnóstico presuntivo de una uveítis intermedia y/o posterior. Igualmente se deben descartar causas infecciosas y autoinmunes, sin embargo el manejo con corticoides tópicos no es recomendado, el médico no oftalmólogo puede iniciar analgesia y cicloplejia antes de la remisión prioritaria al especialista.

Esta revisión sistemática nos permitió evaluar el estado del arte de esta patología, a nivel mundial. Los tratamientos recomendados son esteroides, ciclopléjicos, escopolamina y medicamentos que no se disponen en Colombia, los cuales no tienen evidencia de una mejor respuesta cuando se instauran como tratamiento inicial. La remisión y el tratamiento oportuno de los pacientes con diagnóstico de uveítis son de vital importancia para contribuir a la disminución de secuelas como la catarata y glaucoma además de la pérdida definitiva de la visión. En nuestro medio el primer contacto del paciente suele ser con un médico no especialista o un médico de una especialidad diferente a la oftalmología, en muchas ocasiones la remisión al especialista en oftalmología es demorada o entorpecida por temas administrativos. Por esto, es importante entender 
el rol del médico que tenga el primer contacto con un paciente que presente signos y síntomas de uveítis pues no hay necesidad de equipos especializados para realizar el diagnóstico e iniciar un tratamiento precoz que puede determinar el pronóstico visual de este paciente. Con el propósito de dar respuesta a las inquietudes existentes sobre el abordaje inicial de un paciente con uveítis, por el personal de salud no oftalmólogo, se diseñó un algoritmo de signos y síntomas (anexo 1) que se puedan relacionar con el diagnóstico sin que se requiera el uso de equipos oftalmológicos sofisticados.

\section{Anexos}

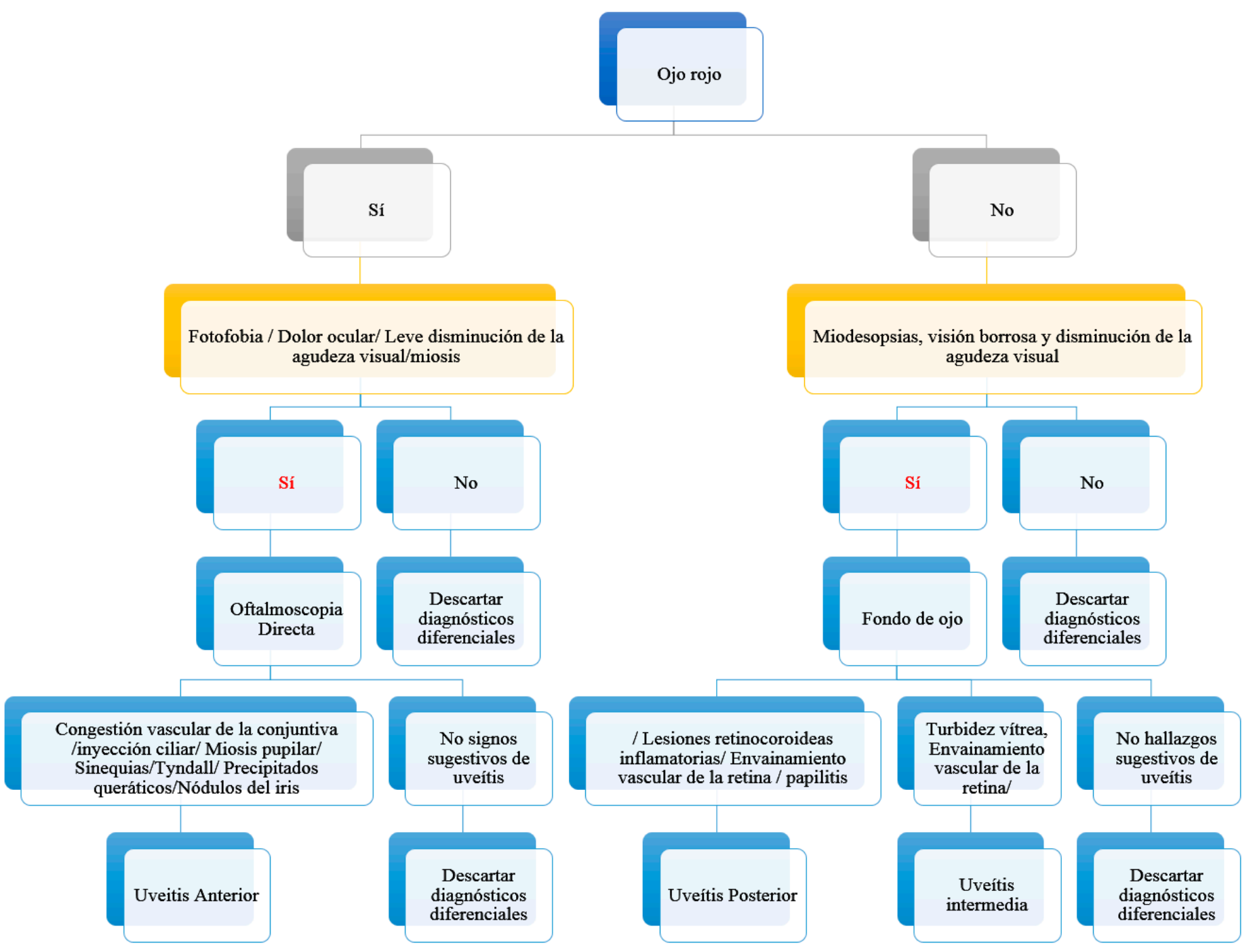




\section{Recomendaciones guía SUN modificada(53)}

\begin{tabular}{|c|c|c|}
\hline \multicolumn{3}{|c|}{ Clasificación Anatómica de la uveítis } \\
\hline TIPO & $\begin{array}{l}\text { SITIO PRIMARIO DE } \\
\text { INFLAMACION }\end{array}$ & INCLUYE \\
\hline $\begin{array}{l}\text { Uveítis Anterior } \\
\text { Uveítis Intermedia } \\
\text { Uveítis posterior }\end{array}$ & $\begin{array}{l}\text { Cámara Anterior } \\
\text { Vitreo } \\
\text { Retina/Coroides }\end{array}$ & $\begin{array}{l}\text { Iritis e iridociclitis } \\
\text { Parsplanitis } \\
\text { Coroiditis: Focal-multifocal, difusa, } \\
\text { coroidorretinitis, retinocoroiditis, } \\
\text { retinitis, neurorretinitis }\end{array}$ \\
\hline Panuveítis & $\begin{array}{l}\text { Cámara anterior. Vitreo. Retina y } \\
\text { coroides }\end{array}$ & \\
\hline \multicolumn{3}{|c|}{ Definición de inicio-duración y curso de la uveitis por el grupo $S U N$} \\
\hline $\begin{array}{l}\text { Categoría } \\
\text { Inicio } \\
\text { Duración }\end{array}$ & $\begin{array}{l}\text { Descripción } \\
\text { Súbito, insidioso } \\
\text { Limitada }<3 \text { meses } \\
\text { Persistente }>3 \text { meses }\end{array}$ & Comentario \\
\hline \multirow[t]{3}{*}{ Curso } & Agudo caracterizado & Súbito y limitado \\
\hline & Recurrente & $\begin{array}{l}\text { Episodios repetidos separados por } \\
\text { episodios de inactividad sin } \\
\text { tratamiento de } 3 \text { meses de duración }\end{array}$ \\
\hline & Crónico & $\begin{array}{l}\text { Uveítis persistente Recurrencia en los } \\
3 \text { meses después de finalizar el } \\
\text { tratamiento }\end{array}$ \\
\hline
\end{tabular}

Graduación de opacidad de cámara anterior -valoración con oftalmoscopia directa

Descripción

0 Negativo-sin opacidad en cámara anterior

$1+$ Leve (se observan detalles del iris y cristalino claros)

2+ Moderada (se observan detalles del iris y cristalino levemente opacos precipitados retro-queráticos)

3+ Marcado (detalles iris y cristalino opacos)

4+ Intenso (acuoso fibrinoide o hipopion )

\footnotetext{
Gradación de opacidad vitrea con oftalmoscopia directa

Grado Descripción de hallazgos clínicos

0 Se observa claramente detalles de polo posterior

1 Mínimo: Polo posterior visible

2Moderado: Polo posterior nublado

3 Marcada: Polo posterior difícilmente visible

4 Severa: Fondo de ojo impracticable
}

\section{Actividad de la uveitis - Terminología}

Definición de Términos

Inactiva Grado 0 sin celularidad

Empeoramiento de la actividad Dos niveles de incremento de la inflamación ejm: células en cámara anterior más opacidad vítrea) o incremento del grado $3+$ a $4+$

Mejoría de la actividad Dos niveles de disminución de inflamación ejm : disminución de células en cámara anterior o en opacidad vítrea o disminución a grado 0

Remisión: Enfermedad Inactiva $>3$ meses después de descontinuar todos los tratamiento para la enfermedad ocular. 


\section{Agradecimientos}

\author{
Dr Luis Jorge Hernández \\ MD, Salud pública y profesor Universidad de Los An- \\ des
}

\section{Luis Jesús Vásquez \\ Médico. Universidad de Los Andes}

\author{
Juanita Valcárcel \\ Médico. Universidad del Rosario
}

\section{Andrea Naranjo \\ Médico Interno. Universidad de Los Andes}

Diego Alba

Estudiante de Medicina. Universidad de Los Andes

\section{Camilo Ángel}

Estudiante de Medicina. Universidad de Los Andes

\section{Iván Triana \\ Estudiante de Medicina. Universidad de Los Andes}

\author{
Santiago Rojas Gómez \\ Estudiante de Medicina. Universidad de Los Andes
}

\section{Andrea Cediel \\ Estudiante de Medicina. Universidad de Los Andes}

\section{Ángela Mariño \\ Estudiante de Medicina. Universidad de Los Andes}

\section{Referencias}

1. Alañon FJ, Fernandez J FS. Oftalmología en la atención primaria. 1ra ed. Editorial Alcala, editor. Madrid; 2001. 177-198 p.

2. Tasman W, Jaeger. EA. Duane's Ophthalmology. Journal of Chemical Information and Modeling. 2009.

3. Yanoff M, Duker J. Opthalmology. Mechanisms of Uveitis. 2nd ed. Edinburgo: Elsevier; 2009. 778-780 p.
4. Borges S. Oftalmología [Internet]. 1 ed La habana. [cited 2012 Mar 8]. Available from: http://www.hospitalameijeiras.sld.cu/hha/mpm/documentos/OFTALMOLOGIA/PA/ UVEITIS.pdf

5. Rathinam SR, Namperumalsamy P. Global variation and pattern changes in epidemiology of uveitis. Indian J Ophthalmol [Internet]. 2007;55(3):173-83. Available from: http://www. ijo.in/article. asp? issn=0301-4738; year $=2007$; volume $=55$;issue $=3 ;$ spage $=173$; epage $=183$; aulast $=$ Rathinam $\backslash$ nhttp $: /$ www . ncbi.nlm.nih.gov/pubmed/17456933

6. De-la-Torre A, Lopez-Castillo CA, Rueda JC, Mantilla RD, Gomez-Marin JE, Anaya JM. Clinical patterns of uveitis in two ophthalmology centres in Bogota, Colombia. Clin Exp Ophthalmol. 2009;37(5):458-66.

7. Yanoff M, Duker J. Anatomy of the Uvea. Edinburgo: Elsevier; 2009. 775-776 p.

8. Foxman EFE, Zhang M, Hurst SD, Muchamuel T, Shen D, Wawrousek EF, et al. Inflammatory mediators in uveitis: differential induction of cytokines and chemokines in Th1- versus Th2-mediated ocular inflammation. J Immunol [Internet]. 2002;168(5):2483-92. Available from: http://www.jimmunol.org/content/168/5/2483.short \nhttp://www.ncbi.nlm. nih.gov/pubmed/11859142

9. Li Z, Lim WK, Mahesh SP, Liu B, Nussenblatt RB. Cutting edge: in vivo blockade of human IL-2 receptor induces expansion of CD 56(bright) regulatory NK cells in patients with active uveitis. J Immunol [Internet]. 2005;174(9):5187-91. Available from: http://www.ncbi.nlm.nih.gov/pubmed/15843513

10. Chan CC, Li Q. Immunopathology of uveitis. Br J Ophthalmol [Internet]. 1998 Jan [cited 2016 Sep 8];82(1):91-6. Available from: http://www.ncbi.nlm.nih.gov/pubmed/9536890

11. Whitcup SM, Chan CC, Li Q, Nussenblatt RB. Expression of cell adhesion molecules in posterior uveitis. Arch Ophthalmol (Chicago, Ill 1960) [Internet]. 1992 May [cited 2016 Sep 8];110(5):662-6. Available from: http://www.ncbi.nlm.nih. gov/pubmed/1374609

12. Chan C-C, Gery I. Mechanisms of uveitis. 3rd ed. Elsevier; 1992.

13. Rosa, JR R. 2015-2016 Basic and Clinical Science Course (bcsc): Ophthalmic Pathology and Intraocular Tumors. Section 4. Ophthalmology AA of, editor. 2015.

14. Zhou R, Caspi RR. Ocular immune privilege. F1000 Biol Rep [Internet]. 2010 [cited 2016 Sep 8];2. Available from: http:// www.ncbi.nlm.nih.gov/pubmed/20948803

15. Taylor AW, Kaplan HJ. Ocular immune privilege in the year 2010: ocular immune privilege and uveitis. Ocul Immunol Inflamm [Internet]. 2010 Dec [cited 2016 Sep 8];18(6):48892. Available from: http://www.ncbi.nlm.nih.gov/pub$\mathrm{med} / 21091058$

16. Smith JR, Jabs DA, Briceland DJ, Holland GN. Education in the ophthalmic discipline of uveitis. Am J Ophthalmol [Internet]. 2008 Dec [cited 2016 Sep 8];146(6):799-801. Available from: http://www.ncbi.nlm.nih.gov/pubmed/19027419 
17. Nussenblatt RB, Whitcup SM. Uveitis: Fundamentals and Clinical Practice. 4rd ed. Chicago: Elsevier; 2010.

18. FT K. Uveitis Infecciosa: Una Revisión. Arch Soc Esp Oftalmol [Internet]. 2000; Available from: http://www.oftalmo. $\mathrm{com} / \mathrm{seo} / 2000 / 04 \mathrm{abr} 00 / 04 . \mathrm{htm}$

19. McCluskey PJ, Towler HM, Lightman S. Management of chronic uveitis. BMJ [Internet]. 2000 Feb 26 [cited 2016 Sep 8];320(7234):555-8. Available from: http://www.ncbi.nlm. nih.gov/pubmed/10688564

20. De Smet MD, Taylor SRJ, Bodaghi B, Miserocchi E, Murray PI, Pleyer U, et al. Understanding uveitis: The impact of research on visual outcomes. Prog Retin Eye Res [Internet]. 2011;30(6):452-70. Available from: http://dx.doi.org/10.1016/j.preteyeres.2011.06.005

21. Herbort CP. Uveitis update: An overview by the society for Ophthalmo-Immunoinfectiology in Europe. Middle East Afr J Ophthalmol [Internet]. 2009 Oct [cited 2016 Sep 8];16(4):157-8. Available from: http://www.ncbi.nlm.nih. gov/pubmed/20404983

22. Chipont BE, Cunningham E. Manejo del paciente con uveítis anterior aguda. Arch Soc Esp Oftalmol [Internet]. 2002;4. Available from: http://www.oftalmo.com/seo/2002/04abr02/04.htm

23. Weinberg R. Anterior uveitis. Ophtalmol clin North Am. 1993;6:23-8.

24. LeHoang P. The gold standard of noninfectious uveitis: Corticosteroids. New Treat Noninfectious Uveitis. 2012;51:7-28.

25. Gomes Bittencourt M, Sepah YJ, Do D V, Agbedia O, Akh$\operatorname{tar} \mathrm{A}$, Liu H, et al. New treatment options for noninfectious uveitis. Dev Ophthalmol [Internet]. 2012 [cited 2016 Sep 8];51:134-61. Available from: http://www.ncbi.nlm.nih.gov/ pubmed/22517211

26. Durrani OM, Meads CA, Murray PI. Uveitis: a potentially blinding disease. Ophthalmol J Int d'ophtalmologie Int J Ophthalmol Zeitschrift für Augenheilkd [Internet]. [cited 2016 Sep 8];218(4):223-36. Available from: http://www. ncbi.nlm.nih.gov/pubmed/15258410

27. Pato E, Muñoz-Fernández S, Francisco F, Abad MA, Maese J, Ortiz A, et al. Systematic review on the effectiveness of immunosuppressants and biological therapies in the treatment of autoimmune posterior uveitis. Semin Arthritis Rheum [Internet]. 2011 Feb [cited 2016 Sep 8];40(4):314-23. Available from: http://www.ncbi.nlm.nih.gov/pubmed/20656330

28. Jabs DA, Rosenbaum JT, Foster CS, Holland GN, Jaffe GJ, Louie JS, et al. Guidelines for the use of immunosuppressive drugs in patients with ocular inflammatory disorders: recommendations of an expert panel. Am J Ophthalmol [Internet]. 2000 Oct [cited 2016 Sep 8];130(4):492-513. Available from: http://www.ncbi.nlm.nih.gov/pubmed/11024423

29. Larson T, Nussenblatt RB, Sen HN. Emerging drugs for uveitis. Expert Opin Emerg Drugs [Internet]. 2011 Jun [cited 2016 Sep 8];16(2):309-22. Available from: http://www.ncbi. nlm.nih.gov/pubmed/21210752
30. Hedayatfar A, Hashemi H, Asgari S, Chee S-P. Comparison of efficacy and ocular surface toxicity of topical preservative-free methylprednisolone and preserved prednisolone in the treatment of acute anterior uveitis. Cornea. 2014;33(4):366-72.

31. Gallego-Pinazo R, Dolz-Marco R, Martínez-Castillo S, Arévalo JF, Díaz-Llopis M. Update on the principles and novel local and systemic therapies for the treatment of non-infectious uveitis. Inflamm Allergy Drug Targets [Internet]. 2013;12(October 2015):38-45. Available from: http://www. ncbi.nlm.nih.gov/pubmed/23441991

32. Kruh J, Stephen Foster C. The philosophy of treatment of uveitis: Past, present and future. New Treat Noninfectious Uveitis. 2012;51:1-6.

33. Siddique SS, Shah R, Suelves AM, Foster CS. Road to remission: a comprehensive review of therapy in uveitis. Expert Opin Investig Drugs [Internet]. 2011;20(11):1497-515. Available from: http://www.ncbi.nlm.nih.gov/pubmed/21936708

34. Lee K, Bajwa A, Freitas-Neto CA, Metzinger JL, Wentworth BA, Foster CS. A comprehensive review and update on the biologic treatment of adult noninfectious uveitis: Part II. Expert Opin Biol Ther [Internet]. 2014;14(11):1651-66. Available from: http://www.embase.com/search/results?subaction $=$ viewrecord $\&$ from $=$ export \&id=L600509390 \nhttp:// dx.doi.org/10.1517/14712598.2014.947957\nhttp://sfx. library.uu.nl/utrecht?sid=EMBASE \&issn=17447682\&id=doi:10.1517/14712598.2014.947957\&atitle=A+comprehensive+rev

35. Pleyer U, Stübiger N. New pharmacotherapy options for noninfectious posterior uveitis. Expert Opin Biol Ther [Internet]. 2014;14(12):1783-99. Available from: http://www.scopus. com/inward/record.url?eid=2-s2.0-84910074151\&partnerI$\mathrm{D}=\mathrm{tZOtx} 3 \mathrm{y} 1$

36. Dhoot DS, Martin DF, Srivastava SK. Pediatric infectious posterior uveitis. Int Ophthalmol Clin [Internet]. 2011;51(1):113-28. Available from: http://www.ncbi.nlm. nih.gov/pubmed/21139480

37. Mandelcorn ED. Infectious causes of posterioruveitis. Can J Ophthalmol [Internet]. 2013;48(1):31-9. Available from: http://dx.doi.org/10.1016/j.jcjo.2012.11.013

38. Jap A, Chee S-P. Viral anterior uveitis. Curr Opin Ophthalmol. 2011;22(6):483-8.

39. Barisani-Asenbauer T, Maca SM, Mejdoubi L, Emminger W, Machold K, Auer H. Uveitis- a rare disease often associated with systemic diseases and infections- a systematic review of 2619 patients. Orphanet J Rare Dis. 2012;7(1):57.

40. Camilo ENR, Moura GL, Arantes TE, \&ordf, e nio F. Clinical and epidemiological characteristics of patients with uveitis in an emergency eye care center in Brazil^ien; Caracter\&Atilde;\&shy;sticas cl\&Atilde;\&shy;nicas e epidemiol\&Atilde;\&sup3;gicas das uve\&Atilde;\&shy;tes em um servi\&Atilde;\&sect;o de urg\&. Arq Bras Oftalmol [Internet]. 2014;2(1):3. Available from: http://www.scielo.br/scielo.php?script=sci_arttext\&amplnpid=S0004-2749201400010003

41. Pan J, Kapur M, McCallum R. Noninfectious immune-me- 
diated uveitis and ocular inflammation. Curr Allergy Asthma Rep. 2014;14(1):1-8.

42. Velu J, Agarwal S, Gupta V, Sharma K, Sharma A, Gupta A. Hypopyon uveitis-a rare presentation of intraocular tuberculosis. Ocul Immunol Inflamm [Internet]. 2013;21(3):2513. Available from: http://www.ncbi.nlm.nih.gov/pub$\operatorname{med} / 23617801$

43. Cohen AE, Assang C, Patane MA, From S, Korenfeld M. Evaluation of dexamethasone phosphate delivered by ocular iontophoresis for treating noninfectious anterior uveitis. Ophthalmology [Internet]. 2012;119(1):66-73. Available from: http://dx.doi.org/10.1016/j.ophtha.2011.07.006

44. Sheppard JD, Toyos MM, Kempen JH, Kaur P, Foster CS. Difluprednate $0.05 \%$ versus prednisolone acetate $1 \%$ for endogenous anterior uveitis: A phase III, multicenter, randomized study. Investig Ophthalmol Vis Sci. 2014;55(5):2993-3002.

45. Kempen JH, Altaweel MM, Holbrook JT, Jabs DA, Louis TA, Sugar EA, et al. Randomized comparison of systemic anti-inflammatory therapy versus fluocinolone acetonide implant for intermediate, posterior, and panuveitis: The multicenter uveitis steroid treatment trial. Ophthalmology. 2011;118(10):1916-26.

46. Selmi C. Diagnosis and classification of autoimmune uveitis. Autoimmun Rev. 2014;13(4-5):591-4.

47. Khairallah M, Kahloun R, Ben Yahia S, Jelliti B, Messaoud R. New infectious etiologies for posterior uveitis. Ophthalmic Res. 2013;49(2):66-72.

48. De Smet MD, Taylor SRJ, Bodaghi B, Miserocchi E, Murray PI, Pleyer U, et al. Understanding uveitis: the impact of research on visual outcomes. Prog Retin Eye Res [Internet]. 2011 Nov [cited 2016 Sep 8];30(6):452-70. Available from: http://www.ncbi.nlm.nih.gov/pubmed/21807112

49. Srivastava A, Rajappa M, Kaur J. Uveitis: Mechanisms and recent advances in therapy. Clin Chim Acta [Internet]. 2010;411(17-18):1165-71. Available from: http://dx.doi.org/10.1016/j.cca.2010.04.017

50. Babu K, Mahendradas P. Medical management of uveitis - current trends. Indian J Ophthalmol [Internet]. 2013;61(6):277-83. Available from: http://www.pubmedcentral.nih.gov/articlerender.fcgi?artid=3744780\&tool=pmcentrez\&rendertype $=$ abstract

51. Majumder PD, Sudharshan S, Biswas J. Laboratory support in the diagnosis of uveitis. Indian J Ophthalmol [Internet]. 2013;61(6):269-76. Available from: http://www.pubmedcentral.nih.gov/articlerender.fcgi? artid=3744779\&tool=pmcentrez\&rendertype $=$ abstract

52. Kim SJ. Diagnosis and management of noninfectious pediatric uveitis. Int Ophthalmol Clin. 2011;51(1):129-45.

53. Zierhut M, Deuter C, Murray PI. Classification of Uveitis Current Guidelines. Eur Ophthalmic Rev [Internet]. 2007 [cited 2016 Sep 20];0(0):77. Available from: http://www. touchophthalmology.com/articles/classification-uveitis-current-guidelines
54. Nausa, J. G. Evaluación Clínica y radiográfica de injertos biocerámicos tipo Hidroxiapatita como alternativa en la reconstrucción de alveolos dentarios postexodoncia. NOVA. 2014; $12(22)$.

55. Flórez, R. A. N. Avances y perspectivas en Síndrome de Asperger. 2014; Nova, 12(21).

56. Rodríguez, O. E., Andrade, W. A., Díaz, F. E., \& Moncada, B. Actividad antimicrobiana de líquenes de la cuenca alta del rio Bogotá. 2015; Nova, 13(23). 